OPEN ACCESS

Edited by:

Daryl Preece,

University of California, San Diego,

United States

Reviewed by:

Ron Orbach,

Yale University, United States

Seok-Cheol Hong,

Korea University, South Korea

${ }^{*}$ Correspondence:

Nancy R. Forde

nforde@sfu.ca

Specialty section:

This article was submitted to

Nanobiotechnology,

a section of the journa

Frontiers in Molecular Biosciences

Received: 29 June 2020

Accepted: 27 August 2020

Published: 06 October 2020

Citation:

Lehmann K, Shayegan M,

Blab GA and Forde NR (2020) Optical

Tweezers Approaches for Probing

Multiscale Protein Mechanics

and Assembly.

Front. Mol. Biosci. 7:577314.

doi: 10.3389/fmolb.2020.577314

\section{Optical Tweezers Approaches for Probing Multiscale Protein Mechanics and Assembly}

\author{
Kathrin Lehmann ${ }^{1,2}$, Marjan Shayegan ${ }^{3}$, Gerhard A. Blab ${ }^{2}$ and Nancy R. Forde ${ }^{1,4,5,6 *}$ \\ 'Department of Physics, Simon Fraser University, Burnaby, BC, Canada, ${ }^{2}$ Soft Condensed Matter and Biophysics, Utrecht \\ University, Utrecht, Netherlands, ${ }^{3}$ School of Engineering and Applied Sciences, Harvard University, Cambridge, MA, \\ United States, ${ }^{4}$ Department of Molecular Biology and Biochemistry, Simon Fraser University, Burnaby, BC, Canada, \\ ${ }^{5}$ Department of Chemistry, Simon Fraser University, Burnaby, BC, Canada, ${ }^{6}$ Centre for Cell Biology, Development \\ and Disease (C2D2), Simon Fraser University, Burnaby, BC, Canada
}

Multi-step assembly of individual protein building blocks is key to the formation of essential higher-order structures inside and outside of cells. Optical tweezers is a technique well suited to investigate the mechanics and dynamics of these structures at a variety of size scales. In this mini-review, we highlight experiments that have used optical tweezers to investigate protein assembly and mechanics, with a focus on the extracellular matrix protein collagen. These examples demonstrate how optical tweezers can be used to study mechanics across length scales, ranging from the single-molecule level to fibrils to protein networks. We discuss challenges in experimental design and interpretation, opportunities for integration with other experimental modalities, and applications of optical tweezers to current questions in protein mechanics and assembly.

Keywords: optical tweezers (OT), protein mechanics, single molecule, microrheology, collagen, protein structure/folding, protein assemblies, fibrillar proteins

\section{INTRODUCTION}

Biology has evolved proteins capable of self-assembly that create dynamic scaffolds imparting mechanical stability and force responsiveness inside and outside of cells. Intracellular proteins include actin, tubulin, tropomyosin and titin, which contribute to cytoskeletal and muscle structure and mechanics. Extracellularly, proteins including collagen, elastin and fibrin assemble to form the extracellular matrix and connective tissues. Understanding how these higher-order assemblies of proteins achieve their responsive mechanical functions requires the ability to measure their mechanical response in different chemical environments and at different hierarchical levels of organization. Because the properties of these proteins are encoded at the molecular level, and because mechanics of the higher-order assemblies can be drastically altered by molecular changes in composition (e.g., mutations, post-translational modifications, age-related chemical changes or ligand binding) (Blank and Boskey, 2008; Li and Cao, 2010; Panwar et al., 2015; Xu et al., 2017; Sorushanova et al., 2019; Hughes et al., 2020), it is important to characterize mechanical response starting from the single-molecule level.

Various techniques have been developed to perform single-molecule force spectroscopy (SMFS). These include atomic force microscopy (AFM), magnetic tweezers (MT), centrifuge force microscopy (CFM), acoustic force spectroscopy (AFS) and optical tweezers (OT). As described in previous reviews (Neuman and Nagy, 2008; Hinterdorfer and Oijen, 2009; Peterman, 2018), 
these techniques confer distinct advantages, meaning the choice of SMFS technique can be dictated by desire for high spatial, temporal and/or force resolution; force range; high throughput measurements; or application of torque in addition to linear stretching forces. However, only some of these approaches are amenable to characterizing mechanics of higher-order protein structures such as filamentous/fibrillar assemblies and largerscale networks.

OT have the broadest applicability in probing the mechanics of protein assemblies at various hierarchies of scale: they offer advantages of high spatial, temporal and force resolution for SFMS, and the ability to passively and actively probe microscale mechanics at prescribed locations within three-dimensional protein networks and even inside living cells (Arbore et al., 2019; Favre-Bulle Itia et al., 2019). This ability to extract force and displacement information across a wide span of system size scales with a single experimental approach facilitates meaningful comparisons of mechanics of proteins at different levels of assembly.

In this mini-review, we highlight some of the applications of OT to the study of protein mechanics, ranging from singlemolecule investigations of mechanics and unfolding to studies of higher-order fibrils and networks. We provide examples of studies on collagen throughout, and highlight work on other assembling protein systems including actin filaments, microtubules, fibrin and prions. We close with a brief discussion of prospects for future research.

\section{SINGLE-MOLECULE INVESTIGATIONS OF PROTEIN MECHANICS}

Single-molecule force spectroscopy studies of protein mechanics require linking the ends of a protein to larger objects that can be independently manipulated. For OT studies, at least one end is linked to a micron-sized bead, which can be held in the focused laser beam of an optical trap (Figure 1). When stretched by its other end (e.g., via manipulation of a bead held on a movable pipette or in an optical trap, or by moving a glass slide), the displacement of the trapped bead from the focus provides the force applied to stretch, while the separation between the two ends gives the end-to-end extension of the molecule (Bustamante et al., 2020). Thus, the primary read-out from an SMFS study is a force-extension curve (FEC), though other modalities such as constant-force measurements can be used to provide deeper information of folding/unfolding dynamics (Bustamante et al., 2004, 2020; Ritchie and Woodside, 2015). FECs reveal information about the elasticity and flexibility of a protein, changes in its structure - such as unfolding - induced by force, and the timescales on which structural changes occur.

Proteins can exhibit a variety of responses to applied force. An entropic elastic response arises when randomly coiled or bent structures are straightened by an applied force, without changing internal structure or contour length. Here, deformation is reversible on the timescale of SMFS and energy put into straightening is recovered upon relaxation. The FEC is monotonic and can be described by the worm-like chain
(WLC) or freely jointed chain (FJC) model of polymer flexibility (Figure 1A). When proteins structurally deform, the FEC can exhibit sharp features, seen as "sawtooth" characteristics arising from domain unfolding and accompanying release of previously buried polypeptide backbone to the force-bearing region of the chain (Figure 1B). The reversibility of these structural changes can be ascertained by looking for hysteresis between stretch and relaxation curves. When pulling occurs more rapidly than internal equilibration of the protein, the loading-rate dependence of unfolding force provides information about the location and heights of free energy barriers, as outlined in Bustamante et al. (2004). Alternatively to sawtooth signatures of domain unfolding, more gradual changes of contour length may occur, arising from structural distortions along the forcebearing backbone. Such backbone lengthening and structural transitions are exhibited by DNA (Bustamante et al., 1994, 2003; Gross et al., 2011; King et al., 2013), and may also contribute to the force response of collagen, a protein with a 300-nm long triple-helical structure (Figure 1A). Initial OTSMFS measurements fit collagen's FEC with the inextensible WLC model with a persistence length of $\sim 15 \mathrm{~nm}$, describing collagen as a relatively flexible polymer (Sun et al., 2002, 2004; Rezaei et al., 2011; Shayegan et al., 2013). More recently, however, measurements of its flexibility using AFM imaging indicate that it is far less flexible, with a persistence length of $\sim 95 \mathrm{~nm}$ (Rezaei et al., 2018), and low-force structural distortions in collagen have been implicated by a variety of single-molecule approaches, including OT (Wieczorek et al., 2015), MT (Adhikari et al., 2011, 2012; Camp et al., 2011), and CFM (Kirkness and Forde, 2018). A low-force-induced structural lengthening of collagen's triple helix could reconcile the disparity in persistence lengths: by fitting a FEC over variable force ranges, it was found that the WLC persistence length increased significantly as the maximum force used for fitting decreased (Wieczorek et al., 2015). Potential mechanisms for a force-induced "softening" of collagen, which may involve bendtwist coupling (Teng and Hwang, 2014), are described in a recent review (Kirkness et al., 2019).

Challenges arise for interpreting molecular flexibility obtained from OT-SMFS measurements when the molecules' contour length is not significantly longer than the persistence length. In this case, the persistence length extracted from WLC fits may significantly underestimate the polymer's true persistence length, as seen for DNA (Seol et al., 2007; Chen et al., 2009). This underestimation arises from the finite length of the experimental chain: the shorter the chain, the greater the relative importance of contributions from its ends. By tethering the chain by its end to surfaces, the orientations of its ends are restricted, which alters the total conformational entropy of the chain and affects determination of its persistence length (Seol et al., 2007; Chen et al., 2009). In some cases, the "true" persistence length can be obtained by measuring polymers of different contour lengths and extrapolating results to the infinite-length limit. This approach has been used for measurements on short lengths of DNA (Seol et al., 2007; Chen et al., 2009), whose contour length is easily controlled, but it is not as easily generalizable to proteins such as collagen, whose length is biologically regulated and 


\section{A}

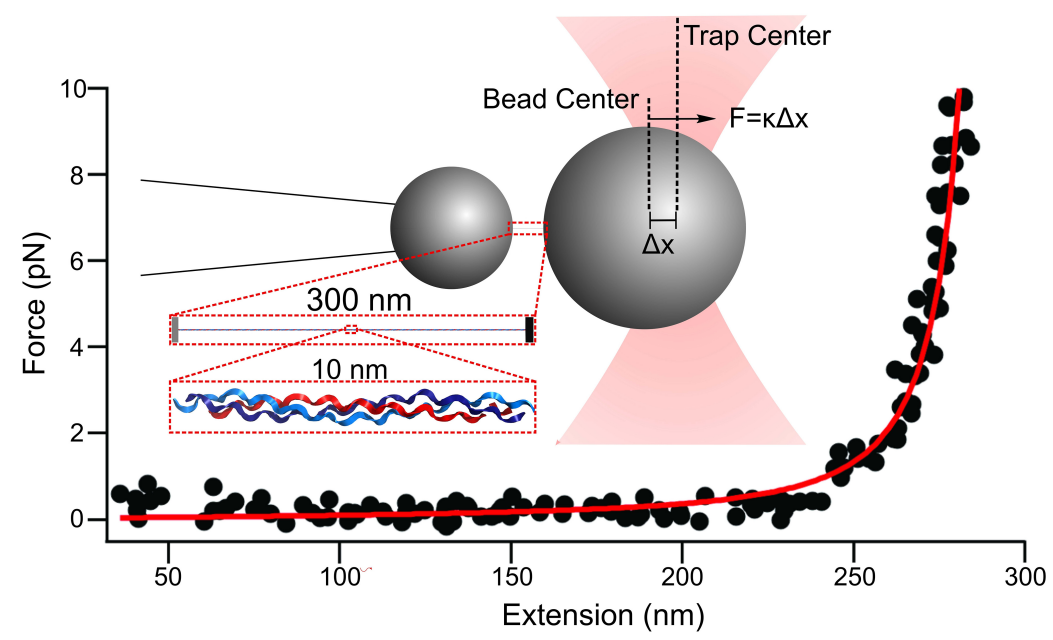

B
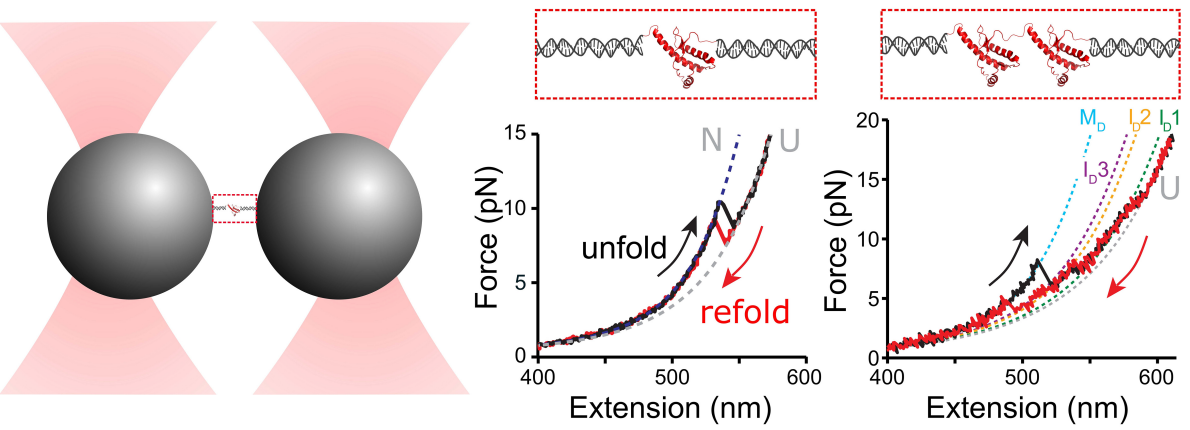

Extension (nm)

Extension (nm)

C

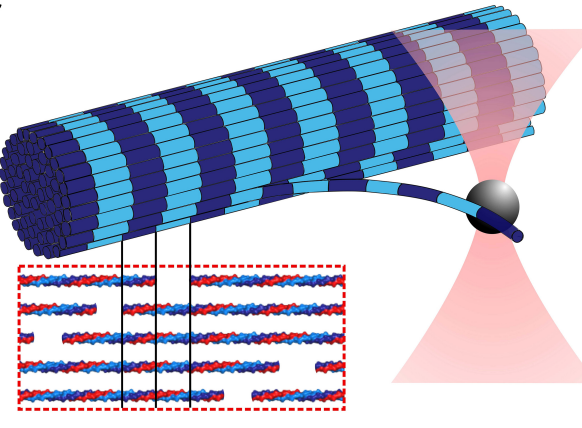

Normal strain

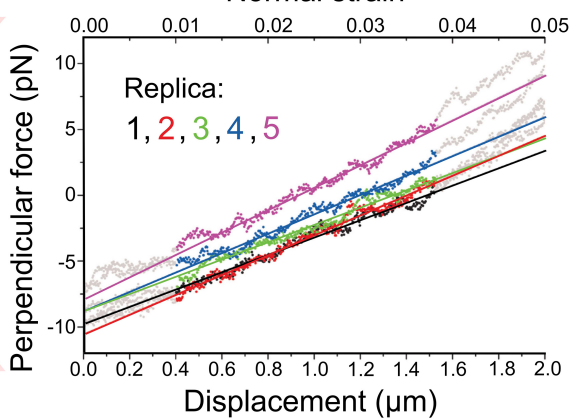

FIGURE 1 | Principles of single-molecule force spectroscopy (SMFS) with optical tweezers (OT). (A) Schematic of an optical tweezers stretching experiment with collagen. The displacement of the bead center from the trap center $\Delta x$ and the trap stiffness $\kappa$ provide the force applied to stretch the molecule. A Worm-Like Chain (WLC) model (red curve) can be used to fit the resulting force extension curve (black dots). Adapted from Wieczorek et al. (2015) with permission. (B) Experimental scheme for OT-SMFS experiments with short proteins. Prion proteins (PrP) are tethered to polystyrene beads via DNA handles (left). The PrP unfolds and refolds to its native state, dependent on the applied force, as a two-state system (middle). PrP dimers linked at their termini lead to complex force extension curves with multiple intermediates, and more remarkably, adopt a misfolded dimer structure at low force rather than two independently folded domains (right). Adapted from Figure 2 in Dee and Woodside (2016) with permission. (C) Illustration of measurements of the bending modulus of a collagen fibril. The inset illustrates the highly ordered lateral organization of collagen molecules within a single fibril, which creates a characteristic "D-banding" pattern (dark/light stripes). The optically trapped bead is used to apply bending deformations to a fibril (left). The resulting force-displacement curve reveals the force required for different applied lateral bending strains (right). Adapted from Dutov et al. (2016) with permission under CC BY license.

which may not fold or be secreted properly if lengthened via genetic engineering.

Probing a tethered molecule substantially shorter than the bead diameter also generates experimental challenges. Having two microspheres at separations much less than their sizes (and the size of the trapping laser focus/foci) can lead to optical interference between a bead and the other trap, an effect which must be deconvolved from the response to obtain the desired force readout of tension applied to the molecule (Downing et al., 2009; Rezaei et al., 2011). Measurements of such short polymers also suffer from amplified effects of stage drift and off-axis stretching. These shortcomings can be addressed by using DNA 
"handles" to link the protein ends to beads, thereby extending the separation between particles and avoiding unwanted optical interference (Figure 1B). DNA handles have become a standard complement of single-molecule measurements of protein folding with optical tweezers (Gosse et al., 2019; Avellaneda et al., 2020). The topic of force-induced protein unfolding, and what can be learned about the energy landscapes, is the subject of many reviews, to which we refer the interested reader (Bustamante et al., 2004, 2020; Ritchie and Woodside, 2015; Hughes and Dougan, 2016; Hoffer and Woodside, 2019).

Optical tweezers-single-molecule force spectroscopy lends itself well to understanding how protein-protein interactions can alter the mechanical landscape. Many proteins retain their structure and independence of folding in the context of neighboring domains - this feature has been used to recapitulate the force response of titin in muscle from studies of its individual domains (Li et al., 2002). Alternatively, protein interaction can stimulate pathological protein misfolding. OT-SMFS studies have demonstrated that prion proteins coupled together in series do not maintain their independent structure, instead adopting new misfolded structures and unfolding pathways compared with monomeric prions (Figure 1B) (Neupane et al., 2014; Dee and Woodside, 2016; Gupta et al., 2016). Because OT can be used to unfold and refold the same protein hundreds to thousands of times, rare folding/unfolding events can be captured, events that may be critical for initiating formation of larger-scale misfolded aggregates that lead to disease (Hoffmann et al., 2013; Yu et al., 2013).

\section{MECHANICS OF HIGHER-ORDER PROTEIN FIBERS AND NETWORKS}

An ongoing challenge is to link protein mechanics at the molecular level to the mechanics of higher-order assemblies. What are the energetic hierarchies governing supramolecular response? In the context of a collagen fibril formed from laterally associated molecules (Figure 1C), for example, what role is played by straightening of collagen's triple-helical backbone (governed by bending rigidity/persistence length at the molecular level) versus molecular deformation (e.g., triple-helix unwinding) versus intermolecular lateral sliding of triple helices? Intermolecular sliding is restricted by covalent crosslinking between collagens, a modification that can be biologically prescribed during assembly and which also can occur, less sitespecifically, as tissues age (Silver et al., 2001; Avery and Bailey, 2008; Depalle et al., 2015; Sorushanova et al., 2019). Crosslinking between chains within a triple helix may also alter its ability to deform at the molecular level when stretched (e.g., by locally pinning and therefore preventing unwinding of the three chains) (Collier et al., 2018; Kirkness and Forde, 2018), though it is less clear how intramolecular crosslinks may affect the bending persistence length at the molecular level (Rezaei et al., 2018; Kirkness et al., 2019). The challenge of linking mechanics at different length scales becomes greater when bridging to even higher-order bundles and networks (e.g., collagen fibrillar gels; Figure 2A) (Gittes and MacKintosh, 1998; Carrillo et al., 2013;
Münster et al., 2013; Broedersz and MacKintosh, 2014; Schnauss et al., 2016; Meng and Terentjev, 2017).

Lateral association into higher-order protein fibers produces structures that are stiffer than their individual protein components. The Young's modulus of such structures is significantly larger than the elastic modulus (proportional to stiffness) of typical optical traps, meaning only high-intensity OT have been used to perform meaningful strain measurements on fibers (Nabiev et al., 2015; Block et al., 2017, 2018; Schepers et al., 2020). A disadvantage of high-intensity optical traps is the local heating of the sample that can result (Neuman and Nagy, 2008). With low-intensity OT, bending moduli of the fibers can be determined by pushing an optically trapped bead laterally against the filament and measuring its deformation as a function of applied force (Figure 1C). OT have been used to measure the bending stiffness of microtubules (Kurachi et al., 1995; Felgner et al., 1996; van Mameren et al., 2009; Koch and Rohrbach, 2018; Memet et al., 2018), actin filaments (van Mameren et al., 2009) and collagen fibrils (Dutov et al., 2016). Other examples of mechanical properties of protein fibers measured with OT include torsional stiffness (Tsuda et al., 1996; Yasuda et al., 1996), bending stiffness of fiber bundles (Rückerl et al., 2017; Strehle et al., 2017), sliding forces of filaments within bundles (Schnauß et al., 2016), and bending and spontaneous assembly of two interacting filaments using a four-trap OT instrument (Kurniawan Nicholas et al., 2016). OT can also be used to measure the assembly and disassembly of individual protein filaments, as has been done for microtubules (Dogterom et al., 2005; Rodríguez-García et al., 2020).

Larger-scale protein networks can be mechanically probed in situ using OT-based microrheology (OT-MR) (Tassieri, 2016; Waigh, 2016; Wei et al., 2017; Robertson-Anderson, 2018). In OT-MR, the motion of an optically trapped bead (constrained within a trap that is either stationary or actively driven) is used to read out information about the frequency-dependent viscoelasticity of its surroundings (Figure 2) (Brau et al., 2007; Mizuno et al., 2008; Waigh, 2016; Robertson-Anderson, 2018). Particle dynamics can be used to determine mechanical properties of the surrounding network. One such property is the complex shear modulus $G^{*}(f)$, comprising the storage and loss moduli $\left[G^{\prime}(f)\right.$ and $G^{\prime \prime}(f)$, respectively]. These moduli describe the elastic and viscous response of the medium (proteins + solvent) surrounding the particle. The frequency dependence of the moduli provides information on the bending rigidities and interactions between protein filaments in solution (Piechocka et al., 2010; Waigh, 2016). Because of this, care must be taken to correctly determine and correct for contributions from OT trap stiffness, which can be particularly difficult when probing softer networks whose elastic modulus is comparable to that of the optical trap (Shayegan and Forde, 2013). Additionally, in situ calibration of optical traps is more challenging in these complex media than in the aqueous solutions used for SFMS, due to the local environment being both viscoelastic and locally heterogeneous, and to light scattering from larger-sized components of the network (Fischer et al., 2010; Hendricks and Goldman, 2017; Staunton et al., 2017). Alternative approaches to MR exist, such as passive observation of bead diffusion, though 

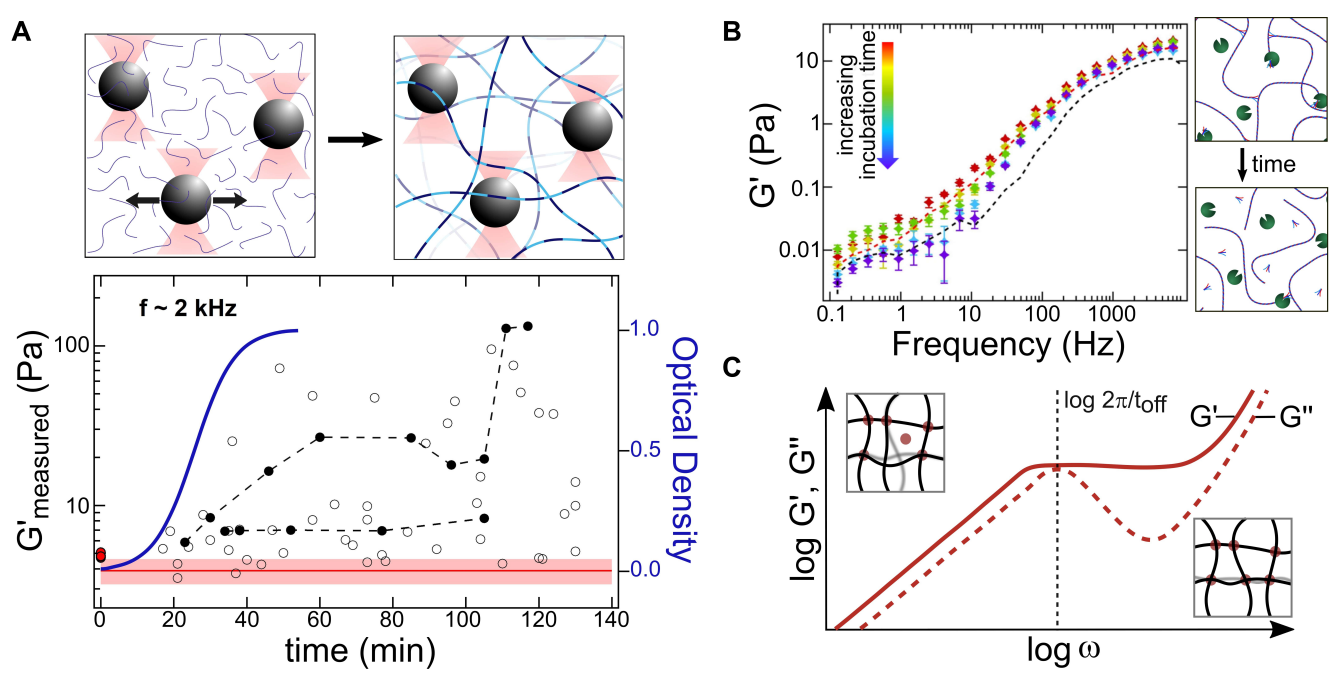

FIGURE 2 | Optical-tweezers based microrheology (OT-MR) measurements of protein network formation and remodeling. (A) Analysis of trapped bead motion is used to determine how the microscale viscoelastic environment changes during assembly and growth of collagen fibrillar networks (schematic upper panel). Arrows indicate thermally driven fluctuations of the particles within the optical traps, used in passive MR experiments to determine the complex shear modulus of the surroundings. For collagen, it was found that elastic moduli and their spread tend to increase during assembly (lower panel, circles), as the local environment becomes more heterogeneous. The red line and shaded region indicate the range of optical trap elastic moduli $G^{\prime}$ trap measured for this bead size, while the red dots at zero time indicate the elastic moduli of trap + collagen solutions in acidic conditions, where assembly cannot occur. Filled black circles indicate repeated measurements on the same bead at multiple times during assembly, illustrating distinct evolutions of local mechanics. These experiments found $\mathrm{G}^{\prime}$ max (at fixed frequencies) to increase with the same sigmoidal kinetics as the optical turbidity used to measure growth of the network (blue curve). Adapted from (Shayegan and Forde, 2013) with permission under CC BY license. (B) OT-MR probes the effect of transient protein-protein interactions that catalyze protein network assembly. Collagen assembly is accelerated by telopeptides, short regions flanking the triple helix (shown as small forked ends in the schematics). Even in acidic conditions where assembly cannot occur, solutions of collagens with intact telopeptides (red dashed line) exhibit a significantly greater G' at low frequencies than collagens with telopeptides enzymatically removed (purple dashed line). The decrease in G' can be detected in real time, as enzymes gradually remove telopeptides (colored markers), thereby reducing protein-protein interactions. Adapted from Shayegan et al. (2016) with permission from Elsevier. (C) It may also be possible to extract the kinetics of transient crosslinking proteins with OT-MR, as found in bulk rheology experiments on actin. At shorter times/higher frequencies (right inset), only short-range bending fluctuations of the actin filaments can occur, while at longer times/lower frequencies (left inset) actin filaments can undergo larger-scale deformation enabled by unbinding of a crosslinking protein (red circle). Thus, G" of a crosslinked actin gel exhibits a local maximum at a frequency corresponding to the unbinding rate (inversely proportional to the characteristic unbinding time $t_{\text {off }}$ ) of the protein crosslinker. Adapted from Broedersz et al. (2010) with permission (copyright 2010 by the American Physical Society).

these generally have a more limited bandwidth and also rely on microscopic tracer beads (Waigh, 2016). The use of beads for these MR approaches creates some requirements in experimental design. Choice of bead size for MR is important: if a continuum measure of network properties is desired, beads should be at least $3 \mathrm{x}$ the pore size of the network (Robertson-Anderson, 2018). Conversely, smaller beads added to a sample are more easily able to navigate through the pores and their motion can reveal information about the sample's spatial heterogeneity. Beads should be sufficiently dilute in the sample so as not to influence the mechanical properties (e.g., forming a much more rigid beadgel composite structure). It is also essential to characterize and control for non-specific interactions between the bead surface and the protein network, a concern that should be addressed in all types of bead-based measurements on proteins and protein networks (Chae and Furst, 2005; Kirkness et al., 2018). OT-MR has been widely applied to characterize network mechanics of cytoskeletal proteins [including actin (Gittes et al., 1997; Brau et al., 2007; Lee et al., 2010; Grebenkov et al., 2013; Atakhorrami et al., 2014; Gurmessa et al., 2017, 2019; Ricketts et al., 2018, 2019), intermediate filaments (Neckernuss et al., 2015; Paust et al., 2016), and microtubules (Ricketts et al., 2018, 2019)] and of extracellular proteins [including collagen (Latinovic et al., 2010; Shayegan and Forde, 2013; Shayegan et al., 2013; Jones et al., 2015; Staunton et al., 2016) and fibrin (Kotlarchyk et al., 2010, 2011; Piechocka et al., 2010; Domínguez-García et al., 2020)].

Most OT-MR studies analyze the motion of a single trapped bead to learn about its local microenvironment, but multipleparticle OT-MR can also be performed. It provides distinct information about the through-space mechanical coupling of the network by analyzing correlated motion between pairs of beads (Crocker et al., 2000). Often one bead is actively displaced with OT and the motion of other (non-optically trapped) beads in the network is recorded, monitoring for example the amplitude and phase lag of their motion relative to the driven particle, which can be used to determine the mechanical transfer function of the network (Paust et al., 2016; Hendricks and Goldman, 2017). For higher-frequency information, several beads can be optically trapped simultaneously, and their correlated motion determined either through active oscillation of one particle or through passive recording of their thermally driven dynamics in stationary traps (Mizuno et al., 2008). Utilizing traps to position beads at desired locations within the network provides greater control over their separations and orientation with respect to 
the (potentially anisotropic) network (Jones et al., 2015). To our knowledge, a maximum of two traps has thus far been employed simultaneously in OT-MR experiments, though more could be implemented with methods such as holographic optical tweezers (van der Horst and Forde, 2008; Jones et al., 2015; Loosemore and Forde, 2017; Bola et al., 2020).

\section{PROTEIN NETWORK FORMATION AND REMODELING}

The assembly of proteins from solution into larger-scale networks triggers many changes in the local microenvironment (Figure 2A), which can be sensed by optically trapped beads. If beads are comparable to the mean pore size in the network, then measurements of local environment may be highly heterogeneous, with some beads sensing essentially solvent while others - more tightly embedded between network filaments report very high elastic moduli. Studies on collagen assembly into fibrillar networks illustrate the heterogeneous properties sensed by micron-sized beads: following triggering of assembly, the heterogeneity in elastic modulus increases as collagens assemble into fibrils that form networks, eventually reaching a plateau (Latinovic et al., 2010; Shayegan and Forde, 2013). The kinetics of increase in $G^{*}(f)$ matches development of turbidity in the sample, indicating commensurate growth in mechanical protein structures and light-scattering fibrillar structures (Figure 2A) (Shayegan and Forde, 2013). OT-MR has been applied to study the triggered assembly and disassembly of other protein networks, such as actin and fibrin (Gurmessa et al., 2019; Domínguez-García et al., 2020).

During MR measurements, optical tweezers also afford the ability to monitor development of mechanical environment at a given location over time, by repeatedly probing the dynamics of the same particle (Figure 2A). This particular type of measurement presents technical challenges: forces exerted by the assembling proteins can be sufficiently strong to displace the particle from the trap (Shayegan and Forde, 2013); and maintaining the optical trap always-on for long periods of time can result in local heating of the sample, which in turn may alter thermally sensitive assembly kinetics and - for proteins with marginal thermal stability such as collagen - even molecular protein structure (Leikina et al., 2002; Neuman and Nagy, 2008). Nonetheless, such measurements are possible, and have been used to characterize locally evolving mechanics during network formation (Latinovic et al., 2010; Shayegan and Forde, 2013; Domínguez-García et al., 2020).

Transient protein-protein interactions, which can provide nucleation points for higher-order assembly, also can be revealed by OT-MR. As one example, collagen assembly into fibrils is facilitated by short non-triple-helical regions at the ends of the collagen molecule, called telopeptides. OT-MR revealed striking enhancement of the viscoelastic properties of solutions of collagens with their telopeptides intact compared with collagens whose telopeptides had been removed (Figure 2B) (Shayegan et al., 2016). These differences were seen in solution conditions that inhibit lateral assembly into fibrils, suggesting that telopeptides enhance stickiness between collagen chains in a variety of solution conditions, a finding supported by a polymer association model. Furthermore, OT-MR was used kinetically to detect enzymatic alteration of these intermolecular interactions: the viscoelasticity of collagen solutions was found to decrease as enzymes cleaved the telopeptides from the ends of the collagen proteins (Figure 2B). It may also be possible for OT-MR to determine the kinetics of transient protein-protein interactions contributing to network assembly. As predicted and found in bulk rheology studies of actin networks, a local maximum in the frequency-dependent loss modulus, $G^{\prime \prime}(f)$, is observed at a frequency corresponding to the unbinding rate of the crosslinking protein alpha-actinin (Figure 2C) (Broedersz et al., 2010). A maximum in $G^{\prime \prime}(f)$ and corresponding turnover in $G^{\prime}(f)$ is predicted to occur when the network components relax significantly quicker than the unbinding of crosslinking elements (Broedersz et al., 2010), implying that this approach could be applied in OT-MR studies of semiflexible protein networks coupled via transient crosslinks to determine unbinding kinetics.

\section{FUTURE PROSPECTS}

Experimentally, much has been learned about protein mechanics and assembly by using optical tweezers in both SMFS and MR configurations. Alternative arrangements of optical traps have the potential to provide distinct insight into the study of protein interactions and assembly. For example, line optical tweezers could be used to study kinetics of transient proteinprotein interactions important for nucleation of higher-order assembly, by studying colloidal binding kinetics arising from protein bridges (Rogers et al., 2013). The use of holographic optical tweezers capable of positioning and quantifying the response of large numbers of particles could be used to determine how network assembly or remodeling is coupled through space and time. Simultaneous measurements at multiple locations throughout the sample during assembly would also provide mechanical insight into how higher-order structure formation percolates throughout the sample (Tran-Ba et al., 2017; Gurmessa et al., 2019). Development of new particles for manipulation can pave the way to higher-force fiber-stretching measurements without the need for higher laser intensities (Jannasch et al., 2012), mitigating concerns about local sample heating. The recent demonstration that nanoscale particles can be used for picoNewton force measurements in OT (Sudhakar et al., 2020) provides many potential advantages to the study of protein mechanics including faster response times, smaller probe surface area, and access within protein networks that have smaller pore sizes.

Integrating other measurement modalities into optical tweezers are likely to provide a deeper mechanistic understanding of protein mechanics and assembly. The incorporation of confocal fluorescence microscopy into bulk rheometers has enabled studies of network formation, reorganization, and fracture in response to macroscopic strains (Münster et al., 2013; Tran-Ba et al., 2017; Burla et al., 2020); similar fluorescence imaging approaches integrated into OT-MR 
would allow mapping of network changes in response to local perturbations, similar to what has been done using phasecontrast imaging during network assembly (Latinovic et al., 2010). SMFS experiments that incorporate OT and singlemolecule fluorescence detection are providing insight into protein-protein (Avellaneda et al., 2020) and DNA-protein (Newton et al., 2019; Wasserman et al., 2019) interactions, as well as structural force-induced changes of biomolecular structure (Gross et al., 2011; King et al., 2013). Care must be taken when integrating single-molecule fluorescence detection into OT instruments, both to achieve high levels of fluorescence detection sensitivity, and, for short protein substrates, to avoid the desired fluorescence signal being overwhelmed by autofluorescence from the particles used for manipulation (Avellaneda et al., 2020). Integrating microfluidics into OT experiments on protein mechanics allows rapid changes of solution environment, enabling studies of chemically triggered network assembly and disassembly (Gurmessa et al., 2019).

Quantifying and rationalizing the mechanics of proteins at various hierarchical scales is critical in fields including biomaterials design (Wu et al., 2018; Sorushanova et al., 2019), neurodegeneration ( $\mathrm{Yu}$ et al., 2013), active matter (Koenderink et al., 2009; Lansky et al., 2015; Weirich et al., 2019; Gompper et al., 2020), cellular biology - including the rapidly developing

\section{REFERENCES}

Adhikari, A. S., Chai, J., and Dunn, A. R. (2011). Mechanical load induces a 100fold increase in the rate of collagen proteolysis by MMP-1. J. Am. Chem. Soc. 133:1686. doi: 10.1021/ja109972p

Adhikari, A. S., Glassey, E., and Dunn, A. R. (2012). Conformational dynamics accompanying the proteolytic degradation of trimeric collagen I by collagenases. J. Am. Chem. Soc. 134, 13259-13265. doi: 10.1021/ja212170b

Alshareedah, I., Moosa, M. M., Raju, M., Potoyan, D. A., and Banerjee, P. R. (2020). Phase transition of RNA-protein complexes into ordered hollow condensates. Proc. Natl. Acad. Sci. U.S.A. 117, 15650-15658. doi: 10.1073/pnas.1922365117

Arbore, C., Perego, L., Sergides, M., and Capitanio, M. (2019). Probing force in living cells with optical tweezers: from single-molecule mechanics to cell mechanotransduction. Biophys. Rev. 11, 765-782. doi: 10.1007/s12551-01900599-y

Atakhorrami, M., Koenderink, G. H., Palierne, J. F., MacKintosh, F. C., and Schmidt, C. F. (2014). Scale-dependent nonaffine elasticity of semiflexible polymer networks. Phys. Rev. Lett. 112:088101. doi: 10.1103/PhysRevLett.112. 088101

Avellaneda, M. J., Koers, E. J., Minde, D. P., Sunderlikova, V., and Tans, S. J. (2020). Simultaneous sensing and imaging of individual biomolecular complexes enabled by modular DNA-protein coupling. Commun. Chem. 3:20. doi: 10 . 1038/s42004-020-0267-4

Avery, N. C., and Bailey, A. J. (2008). "Restraining cross-links responsible for the mechanical properties of collagen fibers: natural and artificial," in Collagen, ed. P. Fratzl (New York, NY: Springer), 81-110. doi: 10.1007/978-0-387-73906-9_4

Bergeron-Sandoval, L.-P., Heris, H. K., Chang, C., Cornell, C. E., Keller, S. L., François, P., et al. (2018). Endocytosis caused by liquid-liquid phase separation of proteins. bioRxiv [Preprint]. doi: 10.1101/145664

Blank, R. D., and Boskey, A. L. (2008). "Genetic collagen diseases: influence of collagen mutations on structure and mechanical behavior," in Collagen, ed. P. Fratzl (New York, NY: Springer), 447-474. doi: 10.1007/978-0-387-73906-9_16

Block, J., Witt, H., Candelli, A., Danes, J. C., Peterman, E. J. G., Wuite, G. J. L., et al. (2018). Viscoelastic properties of vimentin originate from nonequilibrium conformational changes. Sci. Adv. 4:eaat1161. doi: 10.1126/sciadv.aat1161

Block, J., Witt, H., Candelli, A., Peterman, E. J. G., Wuite, G. J. L., Janshoff, A., et al. (2017). Nonlinear loading-rate-dependent force response of individual field of liquid-liquid phase separation (Bergeron-Sandoval et al., 2018; Jawerth et al., 2018; Kaur et al., 2019; Shayegan et al., 2019; Alshareedah et al., 2020), and mechanobiology (Burla et al., 2019; Mathieu and Manneville, 2019). Optical tweezers are well suited to probing protein mechanics at scales ranging from single molecules to fibers to networks, and, with the integration of complementary measurement modalities, will continue to deliver new insight into the mechanisms by which mechanical responsiveness is imparted by proteins.

\section{AUTHOR CONTRIBUTIONS}

All authors contributed to the writing of the manuscript.

\section{FUNDING}

The authors thank the following organizations for funding: Deutsche Forschungsgemeinschaft (DFG; postdoctoral fellowship to KL; Project ID: 415037474), the Fonds de recherche du Québec - Nature et technologies (FRQNT; postdoctoral fellowship to MS), and the Natural Sciences and Engineering Research Council of Canada (NSERC; Discovery Grant to NRF).

vimentin intermediate filaments to applied strain. Phys. Rev. Lett. 118:048101. doi: 10.1103/PhysRevLett.118.048101

Bola, R., Treptow, D., Marzoa, A., Montes-Usategui, M., and Martín-Badosa, E. (2020). Acousto-holographic optical tweezers. Opt. Lett. 45, 2938-2941. doi: 10.1364/OL.391462

Brau, R. R., Ferrer, J. M., Lee, H., Castro, C. E., Tam, B. K., Tarsa, P. B., et al. (2007). Passive and active microrheology with optical tweezers. J. Opt. A 9, S103-S112. doi: 10.1088/1464-4258/9/8/S01

Broedersz, C. P., Depken, M., Yao, N. Y., Pollak, M. R., Weitz, D. A., and MacKintosh, F. C. (2010). Cross-link-governed dynamics of biopolymer networks. Phys. Rev. Lett. 105:238101. doi: 10.1103/PhysRevLett.105.238101

Broedersz, C. P., and MacKintosh, F. C. (2014). Modeling semiflexible polymer networks. Rev. Mod. Phys. 86, 995-1036. doi: 10.1103/RevModPhys.86.995

Burla, F., Dussi, S., Martinez-Torres, C., Tauber, J., van der Gucht, J., and Koenderink, G. H. (2020). Connectivity and plasticity determine collagen network fracture. Proc. Natl. Acad. Sci. U.S.A. 117, 8326-8334. doi: 10.1073/ pnas. 1920062117

Burla, F., Mulla, Y., Vos, B. E., Aufderhorst-Roberts, A., and Koenderink, G. H. (2019). From mechanical resilience to active material properties in biopolymer networks. Nat. Rev. Phys. 1, 249-263. doi: 10.1038/s42254-019-0036-4

Bustamante, C., Alexander, L., Maciuba, K., and Kaiser, C. M. (2020). Singlemolecule studies of protein folding with optical tweezers. Annu. Rev. Biochem. 89, 443-470. doi: 10.1146/annurev-biochem-013118-111442

Bustamante, C., Bryant, Z., and Smith, S. B. (2003). Ten years of tension: singlemolecule DNA mechanics. Nature 421, 423-427. doi: 10.1038/nature01405

Bustamante, C., Chemla, Y. R., Forde, N. R., and Izhaky, D. (2004). Mechanical processes in biochemistry. Annu. Rev. Biochem. 73, 705-748. doi: 10.1146/ annurev.biochem.72.121801.161542

Bustamante, C., Marko, J. F., Siggia, E. D., and Smith, S. (1994). Entropic elasticity of lambda-phage DNA. Science 265, 1599-1600. doi: 10.1126/science.8079175

Camp, R. J., Liles, M., Beale, J., Saeidi, N., Flynn, B. P., Moore, E., et al. (2011). Molecular mechanochemistry: low force switch slows enzymatic cleavage of human type I collagen monomer. J. Am. Chem. Soc. 133:4073. doi: 10.1021/ ja110098b

Carrillo, J. M. Y., MacKintosh, F. C., and Dobrynin, A. V. (2013). Nonlinear elasticity: from single chain to networks and gels. Macromolecules 46, 36793692. doi: $10.1021 / \mathrm{ma} 400478 \mathrm{f}$ 
Chae, B. S., and Furst, E. M. (2005). Probe surface chemistry dependence and local polymer network structure in F-actin microrheology. Langmuir 21, 3084-3089. doi: $10.1021 / 1 \mathrm{la} 0480890$

Chen, Y.-F., Wilson, D. P., Raghunathan, K., and Meiners, J.-C. (2009). Entropic boundary effects on the elasticity of short DNA molecules. Phys. Rev. E 80:020903. doi: 10.1103/PhysRevE.80.020903

Collier, T. A., Nash, A., Birch, H. L., and de Leeuw, N. H. (2018). Effect on the mechanical properties of type I collagen of intra-molecular lysine-arginine derived advanced glycation end-product cross-linking. J. Biomech. 67, 55-61. doi: 10.1016/j.jbiomech.2017.11.021

Crocker, J. C., Valentine, M. T., Weeks, E. R., Gisler, T., Kaplan, P. D., Yodh, A. G., et al. (2000). Two-point microrheology of inhomogeneous soft materials. Phys. Rev. Lett. 85, 888-891. doi: 10.1103/physrevlett.85.888

Dee, D. R., and Woodside, M. T. (2016). Comparing the energy landscapes for native folding and aggregation of PrP. Prion 10, 207-220. doi: 10.1080/ 19336896.2016.1173297

Depalle, B., Qin, Z., Shefelbine, S. J., and Buehler, M. J. (2015). Influence of crosslink structure, density and mechanical properties in the mesoscale deformation mechanisms of collagen fibrils. J. Mech. Behav. Biomed. Mater. 52, 1-13. doi: 10.1016/j.jmbbm.2014.07.008

Dogterom, M., Kerssemakers, J. W., Romet-Lemonne, G., and Janson, M. E. (2005). Force generation by dynamic microtubules. Curr. Opin. Cell Biol. 17, 67-74. doi: 10.1016/j.ceb.2004.12.011

Domínguez-García, P., Dietler, G., Forró, L., and Jeney, S. (2020). Filamentous and step-like behavior of gelling coarse fibrin networks revealed by high-frequency microrheology. Soft Matter 16, 4234-4242. doi: 10.1039/c9sm02228g

Downing, B. P. B., van der Horst, A., Miao, M., Keeley, F. W., and Forde, N. R. (2009). "Probing the elasticity of short proteins with optical tweezers," in Paper Presented at Advances in Imaging, OSA Technical Digest (Washington, DC: Optical Society of America). doi: 10.1364/OTA.2009.OTuA3

Dutov, P., Antipova, O., Varma, S., Orgel, J., and Schieber, J. D. (2016). Measurement of elastic modulus of collagen type I single fiber. PLoS One 11:e0145711. doi: 10.1371/journal.pone.0145711

Favre-Bulle Itia, A., Stilgoe Alexander, B., Scott Ethan, K., and RubinszteinDunlop, H. (2019). Optical trapping in vivo: theory, practice, and applications. Nanophotonics 8, 1023-1040. doi: 10.1515/nanoph-2019-0055

Felgner, H., Frank, R., and Schliwa, M. (1996). Flexural rigidity of microtubules measured with the use of optical tweezers. J. Cell Sci. 109, 509-516.

Fischer, M., Richardson, A. C., Reihani, S. N. S., Oddershede, L. B., and BergSorensen, K. (2010). Active-passive calibration of optical tweezers in viscoelastic media. Rev. Sci. Instrum. 81:015103. doi: 10.1063/1.3280222

Gittes, F., and MacKintosh, F. C. (1998). Dynamic shear modulus of a semiflexible polymer network. Phys. Rev. E 58, R1241-R1244. doi: 10.1103/PhysRevE.58. R1241

Gittes, F., Schnurr, B., Olmsted, P. D., MacKintosh, F. C., and Schmidt, C. F. (1997). Microscopic viscoelasticity: shear moduli of soft materials determined from thermal fluctuations. Phys. Rev. Lett. 79, 3286-3289. doi: 10.1103/physrevlett. 79.3286

Gompper, G., Winkler, R. G., Speck, T., Solon, A., Nardini, C., Peruani, F., et al. (2020). The 2020 motile active matter roadmap. J. Phys. Condens. Matter 32:193001. doi: 10.1088/1361-648x/ab6348

Gosse, C., Strick, T. R., and Kostrz, D. (2019). Molecular scaffolds: when DNA becomes the hardware for single-molecule investigations. Curr. Opin. Chem. Biol. 53, 192-203. doi: 10.1016/j.cbpa.2019.09.006

Grebenkov, D. S., Vahabi, M., Bertseva, E., Forró, L., and Jeney, S. (2013). Hydrodynamic and subdiffusive motion of tracers in a viscoelastic medium. Phys. Rev. E 88:040701. doi: 10.1103/PhysRevE.88.040701

Gross, P., Laurens, N., Oddershede, L. B., Bockelmann, U., Peterman, E. J. G., and Wuite, G. J. L. (2011). Quantifying how DNA stretches, melts and changes twist under tension. Nat. Phys. 7, 731-736. doi: 10.1038/nphys2002

Gupta, A. N., Neupane, K., Rezajooei, N., Cortez, L. M., Sim, V. L., and Woodside, M. T. (2016). Pharmacological chaperone reshapes the energy landscape for folding and aggregation of the prion protein. Nat. Commun. 7:12058. doi: 10.1038/ncomms12058

Gurmessa, B., Ricketts, S., and Robertson-Anderson, R. M. (2017). Nonlinear actin deformations lead to network stiffening, yielding, and nonuniform stress propagation. Biophys. J. 113, 1540-1550. doi: 10.1016/j.bpj.2017. 01.012
Gurmessa, B. J., Bitten, N., Nguyen, D. T., Saleh, O. A., Ross, J. L., Das, M., et al. (2019). Triggered disassembly and reassembly of actin networks induces rigidity phase transitions. Soft Matter 15, 1335-1344. doi: 10.1039/c8sm01912f

Hendricks, A. G., and Goldman, Y. E. (2017). "Measuring molecular forces using calibrated optical tweezers in living cells," in Optical Tweezers: Methods and Protocols, ed. A. Gennerich (New York, NY: Springer), 537-552. doi: 10.1007/ 978-1-4939-6421-5_21

Hinterdorfer, P., and Oijen, A. (2009). Handbook of Single-Molecule Biophysics. New York, NY: Springer. doi: 10.1007/978-0-387-76497-9

Hoffer, N. Q., and Woodside, M. T. (2019). Probing microscopic conformational dynamics in folding reactions by measuring transition paths. Curr. Opin. Chem. Biol. 53, 68-74. doi: 10.1016/j.cbpa.2019.07.006

Hoffmann, A., Neupane, K., and Woodside, M. T. (2013). Single-molecule assays for investigating protein misfolding and aggregation. Phys. Chem. Chem. Phys. 15, 7934-7948. doi: 10.1039/c3cp44564j

Hughes, M. D. G., Cussons, S., Mahmoudi, N., Brockwell, D. J., and Dougan, L. (2020). Single molecule protein stabilisation translates to macromolecular mechanics of a protein network. Soft Matter 16, 6389-6399. doi: 10.1039/ c9sm02484k

Hughes, M. L., and Dougan, L. (2016). The physics of pulling polyproteins: a review of single molecule force spectroscopy using the AFM to study protein unfolding. Rep. Prog. Phys. 79:076601. doi: 10.1088/0034-4885/79/7/076601

Jannasch, A., Demirörs, A. F., van Oostrum, P. D. J., van Blaaderen, A., and Schäffer, E. (2012). Nanonewton optical force trap employing anti-reflection coated, high-refractive-index titania microspheres. Nat. Photonics 6, 469-473. doi: 10.1038/nphoton.2012.140

Jawerth, L. M., Ijavi, M., Ruer, M., Saha, S., Jahnel, M., Hyman, A. A., et al. (2018). Salt-dependent rheology and surface tension of protein condensates using optical traps. Phys. Rev. Lett. 121:258101. doi: 10.1103/PhysRevLett.121.258101

Jones, C. A. R., Cibula, M., Feng, J. C., Krnacik, E. A., McIntyre, D. H., Levine, H., et al. (2015). Micromechanics of cellularized biopolymer networks. Proc. Natl. Acad. Sci. U.S.A. 112, E5117-E5122. doi: 10.1073/pnas.1509663112

Kaur, T., Alshareedah, I., Wang, W., Ngo, J., Moosa, M. M., and Banerjee, P. R. (2019). Molecular crowding tunes material states of ribonucleoprotein condensates. Biomolecules 9:71. doi: 10.3390/biom9020071

King, G. A., Gross, P., Bockelmann, U., Modesti, M., Wuite, G. J. L., and Peterman, E. J. G. (2013). Revealing the competition between peeled ssDNA, melting bubbles, and S-DNA during DNA overstretching using fluorescence microscopy. Proc. Natl. Acad. Sci. U.S.A. 110, 3859-3864. doi: 10.1073/pnas. 1213676110

Kirkness, M. W. H., and Forde, N. R. (2018). Single-molecule assay for proteolytic susceptibility: force-induced collagen destabilization. Biophys. J. 114, 570-576. doi: 10.1016/j.bpj.2017.12.006

Kirkness, M. W. H., Korosec, C. S., and Forde, N. R. (2018). Modified pluronic F127 Surface for bioconjugation and blocking nonspecific adsorption of microspheres and biomacromolecules. Langmuir 34, 13550-13557. doi: 10 . 1021/acs.langmuir.8b02877

Kirkness, M. W. H., Lehmann, K., and Forde, N. R. (2019). Mechanics and structural stability of the collagen triple helix. Curr. Opin. Chem. Biol. 53, 98-105. doi: 10.1016/j.cbpa.2019.08.001

Koch, M. D., and Rohrbach, A. (2018). Label-free imaging and bending analysis of microtubules by ROCS microscopy and optical trapping. Biophys. J. 114, 168-177. doi: 10.1016/j.bpj.2017.10.036

Koenderink, G. H., Dogic, Z., Nakamura, F., Bendix, P. M., MacKintosh, F. C., Hartwig, J. H., et al. (2009). An active biopolymer network controlled by molecular motors. Proc. Natl. Acad. Sci. U.S.A. 106, 15192-15197. doi: 10.1073/ pnas.0903974106

Kotlarchyk, M. A., Botvinick, E. L., and Putnam, A. J. (2010). Characterization of hydrogel microstructure using laser tweezers particle tracking and confocal reflection imaging. J. Phys. Condens. Matter 22:194121. doi: 10.1088/09538984/22/19/194121

Kotlarchyk, M. A., Shreim, S. G., Alvarez-Elizondo, M. B., Estrada, L. C., Singh, R., Valdevit, L., et al. (2011). Concentration independent modulation of local micromechanics in a fibrin gel. PLoS One 6:e20201. doi: 10.1371/journal.pone. 0020201

Kurachi, M., Hoshi, M., and Tashiro, H. (1995). Buckling of a single microtubule by optical trapping forces - direct measurement of microtubule rigidity. Cell Motil. Cytoskeleton 30, 221-228. doi: 10.1002/cm.970300306 
Kurniawan Nicholas, A., Vos Bart, E., Biebricher, A., Wuite Gijs, J. L., Peterman Erwin, J. G., and Koenderink Gijsje, H. (2016). Fibrin networks support recurring mechanical loads by adapting their structure across multiple scales. Biophys. J. 111, 1026-1034. doi: 10.1016/j.bpj.2016.06.034

Lansky, Z., Braun, M., Lüdecke, A., Schlierf, M., ten Wolde, Pieter R, Janson, Marcel $\mathrm{E}$, et al. (2015). Diffusible crosslinkers generate directed forces in microtubule networks. Cell 160, 1159-1168. doi: 10.1016/j.cell.2015.01.051

Latinovic, O., Hough, L. A., and Ou-Yang, H. D. (2010). Structural and micromechanical characterization of type I collagen gels. J. Biomech. 43, 500505. doi: 10.1016/j.jbiomech.2009.09.041

Lee, H., Ferrer, J. M., Nakamura, F., Lang, M. J., and Kamm, R. D. (2010). Passive and active microrheology for cross-linked F-actin networks in vitro. Acta Biomater. 6, 1207-1218. doi: 10.1016/j.actbio.2009.10.044

Leikina, E., Mertts, M. V., Kuznetsova, N., and Leikin, S. (2002). Type I collagen is thermally unstable at body temperature. Proc. Natl. Acad. Sci. U.S.A. 99, 1314-1318. doi: $10.1073 /$ pnas.032307099

Li, H., and Cao, Y. (2010). Protein mechanics: from single molecules to functional biomaterials. Acc. Chem. Res. 43, 1331-1341. doi: 10.1021/ar100057a

Li, H., Linke, W. A., Oberhauser, A. F., Carrion-Vazquez, M., Kerkvliet, J. G., Lu, H., et al. (2002). Reverse engineering of the giant muscle protein titin. Nature 418, 998-1002. doi: 10.1038/nature00938

Loosemore, V. E., and Forde, N. R. (2017). Effects of finite and discrete sampling and blur on microrheology experiments. Opt. Express 25, 31239-31252. doi: 10.1364/OE.25.031239

Mathieu, S., and Manneville, J.-B. (2019). Intracellular mechanics: connecting rheology and mechanotransduction. Curr. Opin. Cell Biol. 56, 34-44. doi: 10. 1016/j.ceb.2018.08.007

Memet, E., Hilitski, F., Morris, M. A., Schwenger, W. J., Dogic, Z., and Mahadevan, L. (2018). Microtubules soften due to cross-sectional flattening. Elife 7:e34695. doi: $10.7554 /$ eLife.34695.023

Meng, F. L., and Terentjev, E. M. (2017). Theory of semiflexible filaments and networks. Polymers 9, 52. doi: 10.3390/polym 9020052

Mizuno, D., Head, D. A., MacKintosh, F. C., and Schmidt, C. F. (2008). Active and passive microrheology in equilibrium and nonequilibrium systems. Macromolecules 41, 7194-7202. doi: 10.1021/ma801218z

Münster, S., Jawerth, L. M., Leslie, B. A., Weitz, J. I., Fabry, B., and Weitz, D. A. (2013). Strain history dependence of the nonlinear stress response of fibrin and collagen networks. Proc. Natl. Acad. Sci. U.S.A. 110, 12197-12202. doi: $10.1073 /$ pnas. 1222787110

Nabiev, S. R., Ovsyannikov, D. A., Kopylova, G. V., Shchepkin, D. V., Matyushenko, A. M., Koubassova, N. A., et al. (2015). Stabilizing the central part of tropomyosin increases the bending stiffness of the thin filament. Biophys. J. 109, 373-379. doi: 10.1016/j.bpj.2015.06.006

Neckernuss, T., Mertens, L. K., Martin, I., Paust, T., Beil, M., and Marti, O. (2015). Active microrheology with optical tweezers: a versatile tool to investigate anisotropies in intermediate filament networks. J. Phys. D Appl. Phys. 49:045401. doi: 10.1088/0022-3727/49/4/045401

Neuman, K. C., and Nagy, A. (2008). Single-molecule force spectroscopy: optical tweezers, magnetic tweezers and atomic force microscopy. Nat. Methods 5, 491-505. doi: 10.1038/nmeth.1218

Neupane, K., Solanki, A., Sosova, I., Belov, M., and Woodside, M. T. (2014). Diverse metastable structures formed by small oligomers of $\alpha$-synuclein probed by force spectroscopy. PLoS One 9:e86495. doi: 10.1371/journal.pone.0086495

Newton, M. D., Taylor, B. J., Driessen, R. P. C., Roos, L., Cvetesic, N., Allyjaun, S., et al. (2019). DNA stretching induces Cas9 off-target activity. Nat. Struct. Mol. Biol. 26, 185-192. doi: 10.1038/s41594-019-0188-z

Panwar, P., Lamour, G., Mackenzie, N. C. W., Yang, H., Ko, F., Li, H., et al. (2015). Changes in structural-mechanical properties and degradability of collagen during aging-associated modifications. J. Biol. Chem. 290, 23291-23306. doi: 10.1074/jbc.M115.644310

Paust, T., Neckernuss, T., Mertens, L. K., Martin, I., Beil, M., Walther, P., et al. (2016). Active multi-point microrheology of cytoskeletal networks. Beilstein J. Nanotechnol. 7, 484-491. doi: 10.3762/bjnano.7.42

Peterman, E. J. G. (2018). Single Molecule Techniques: Methods and Protocols, 2nd Edn. New York, NY: Humana Press. doi: 10.1007/978-1-4939-7271-5

Piechocka, I. K., Bacabac, R. G., Potters, M., MacKintosh, F. C., and Koenderink, G. H. (2010). Structural hierarchy governs fibrin gel mechanics. Biophys. J. 98, 2281-2289. doi: 10.1016/j.bpj.2010.01.040
Rezaei, N., Downing, B. P. B., Wieczorek, A., Chan, C. K. Y., Welch, R. L., and Forde, N. R. (2011). "Using optical tweezers to study mechanical properties of collagen," in Proceedings of SPIE Photonics North 2011, eds R. Kashyap, M. Tetu, and R. N. Kleiman (Bellingham, WA: SPIE-Int Soc Optical Engineering). doi: $10.1117 / 12.905714$

Rezaei, N., Lyons, A., and Forde, N. R. (2018). Environmentally controlled curvature of single collagen proteins. Biophys. J. 115, 1457-1469. doi: 10.1016/ j.bpj.2018.09.003

Ricketts, S. N., Francis, M. L., Farhadi, L., Rust, M. J., Das, M., Ross, J. L., et al. (2019). Varying crosslinking motifs drive the mesoscale mechanics of actin-microtubule composites. Sci. Rep. 9:12831. doi: 10.1016/j.bpj.2018.09.003

Ricketts, S. N., Ross, J. L., and Robertson-Anderson, R. M. (2018). Co-entangled actin-microtubule composites exhibit tunable stiffness and power-law stress relaxation. Biophys. J. 115, 1055-1067. doi: 10.1016/j.bpj.2018.08.010

Ritchie, D. B., and Woodside, M. T. (2015). Probing the structural dynamics of proteins and nucleic acids with optical tweezers. Curr. Opin. Struct. Biol. 34, 43-51. doi: 10.1016/j.sbi.2015.06.006

Robertson-Anderson, R. M. (2018). Optical tweezers microrheology: from the basics to advanced techniques and applications. ACS Macro Lett. 7, 968-975. doi: 10.1021/acsmacrolett.8b00498

Rodríguez-García, R., Volkov, V. A., Chen, C.-Y., Katrukha, E. A., Olieric, N., Aher, A., et al. (2020). Mechanisms of motor-independent membrane remodeling driven by dynamic microtubules. Curr. Biol. 30, 972-987. doi: 10.1016/j.cub. 2020.01.036

Rogers, W. B., Sinno, T., and Crocker, J. C. (2013). Kinetics and non-exponential binding of DNA-coated colloids. Soft Matter 9, 6412-6417. doi: 10.1039/ c3sm50593f

Rückerl, F., Lenz, M., Betz, T., Manzi, J., Martiel, J. L., Safouane, M., et al. (2017). Adaptive response of actin bundles under mechanical stress. Biophys. J. 113, 1072-1079. doi: 10.1016/j.bpj.2017.07.017

Schepers, A. V., Lorenz, C., and Köster, S. (2020). Tuning intermediate filament mechanics by variation of $\mathrm{pH}$ and ion charges. Nanoscale 12, 15236-15245. doi: $10.1039 /$ D0NR02778B

Schnauss, J., Handler, T., and Kas, J. A. (2016). Semiflexible Biopolymers in Bundled Arrangements. Polymers 8, 274. doi: 10.3390/polym8080274

Schnauß, J., Golde, T., Schuldt, C., Schmidt, B. U. S., Glaser, M., Strehle, D., et al. (2016). Transition from a linear to a harmonic potential in collective dynamics of a multifilament actin bundle. Phys. Rev. Lett. 116:108102. doi: 10.1103/PhysRevLett.116.108102

Seol, Y., Li, J., Nelson, P. C., Perkins, T. T., and Betterton, M. D. (2007). Elasticity of short DNA molecules: theory and experiment for contour lengths of 0.6-7 $\mu \mathrm{m}$. Biophys. J. 93, 4360-4373. doi: 10.1529/biophysj.107.112995

Shayegan, M., Altindal, T., Kiefl, E., and Forde, N. R. (2016). Intact telopeptides enhance interactions between collagens. Biophys. J. 111, 2404-2416. doi: 10. 1016/j.bpj.2016.10.039

Shayegan, M., and Forde, N. R. (2013). Microrheological characterization of collagen systems: from molecular solutions to fibrillar gels. PLoS One 8:e70590. doi: 10.1371/journal.pone.0070590

Shayegan, M., Rezaei, N., Lam, N. H., Altindal, T., Wieczorek, A., and Forde, N. R. (2013). "Probing multiscale mechanics of collagen with optical tweezers," in Proceedings of SPIE Optical Trapping and Optical Micromanipulation X, eds K. Dholakia and G. C. Spalding (Bellingham, WA: SPIE-Int Soc Optical Engineering). doi: 10.1117/12.2027258

Shayegan, M., Tahvildari, R., Metera, K., Kisley, L., Michnick, S. W., and Leslie, S. R. (2019). Probing inhomogeneous diffusion in the microenvironments of phaseseparated polymers under confinement. J. Am. Chem. Soc. 141, 7751-7757. doi: $10.1021 /$ jacs. 8 b 13349

Silver, F. H., Christiansen, D. L., Snowhill, P. B., and Chen, Y. (2001). Transition from viscous to elastic-based dependency of mechanical properties of selfassembled type I collagen fibers. J. Appl. Polym. Sci. 79, 134-142. doi: 10.1002/ 1097-4628(20010103)79:1<134::AID-APP160>3.0.CO;2-E

Sorushanova, A., Delgado, L. M., Wu, Z., Shologu, N., Kshirsagar, A., Raghunath, R., et al. (2019). The collagen suprafamily: from biosynthesis to advanced biomaterial development. Adv. Mater. 31:e1801651. doi: 10.1002/adma. 201801651

Staunton, J. R., Blehm, B., Devine, A., and Tanner, K. (2017). In situ calibration of position detection in an optical trap for active microrheology in viscous materials. Opt. Express 25, 1746-1761. doi: 10.1364/OE.25.001746 
Staunton, J. R., Vieira, W., Fung, K. L., Lake, R., Devine, A., and Tanner, K. (2016). Mechanical properties of the tumor stromal microenvironment probed in vitro and ex vivo by in situ-calibrated optical trap-based active microrheology. Cell. Mol. Bioeng. 9, 398-417. doi: 10.1007/s12195-016-0460-9

Strehle, D., Mollenkopf, P., Glaser, M., Golde, T., Schuldt, C., Kas, J. A., et al. (2017). Single actin bundle rheology. Molecules 22, 1804. doi: 10.3390/ molecules22101804

Sudhakar, S., Abdosamadi, M. K., Jachowski, T. J., Bugiel, M., Jannasch, A., and Schäffer, E. (2020). Germanium nanospheres for ultraresolution picotensiometry of kinesin motors. bioRxiv [Preprint]. doi: 10.1101/2020.06.18. 159640

Sun, Y. L., Luo, Z. P., Fertala, A., and An, K. N. (2002). Direct quantification of the flexibility of type I collagen monomer. Biochem. Biophys. Res. Commun. 295, 382-386. doi: 10.1016/S0006-291X(02)00685-X

Sun, Y. L., Luo, Z. P., Fertala, A., and An, K. N. (2004). Stretching type II collagen with optical tweezers. J. Biomech. 37, 1665-1669. doi: 10.1016/j.jbiomech.2004. 02.028

Tassieri, M. (2016). Microrheology with Optical Tweezers: Principles and Applications. Boca Raton, FL: CRC Press. doi: 10.1201/9781315364872

Teng, X., and Hwang, W. (2014). Chain registry and load-dependent conformational dynamics of collagen. Biomacromolecules 15, 3019-3029. doi: 10.1021/bm500641f

Tran-Ba, K.-H., Lee, D. J., Zhu, J., Paeng, K., and Kaufman, L. J. (2017). Confocal rheology probes the structure and mechanics of collagen through the sol-gel transition. Biophys. J. 113, 1882-1892. doi: 10.1016/j.bpj.2017.08.025

Tsuda, Y., Yasutake, H., Ishijima, A., and Yanagida, T. (1996). Torsional rigidity of single actin filaments and actin-actin bond breaking force under torsion measured directly by in vitro micromanipulation. Proc. Natl. Acad. Sci. U.S.A. 93, 12937-12942. doi: 10.1073/pnas.93.23.12937

van der Horst, A., and Forde, N. R. (2008). Calibration of dynamic holographic optical tweezers for force measurements on biomaterials. Opt. Express 16, 20987-21003. doi: 10.1364/OE.16.020987

van Mameren, J., Vermeulen, K. C., Gittes, F., and Schmidt, C. F. (2009). Leveraging single protein polymers to measure flexural rigidity. J. Phys. Chem. B 113, 3837-3844. doi: 10.1021/jp808328a

Waigh, T. A. (2016). Advances in the microrheology of complex fluids. Rep. Prog. Phys. 79:074601. doi: 10.1088/0034-4885/79/7/074601
Wasserman, M. R., Schauer, G. D., O’Donnell, M. E., and Liu, S. (2019). Replication fork activation Is enabled by a single-stranded DNA gate in CMG helicase. Cell 178, 600-611. doi: 10.1016/j.cell.2019.06.032

Wei, M.-T., Latinovic, O., Hough, L. A., Chen, Y.-Q., Ou-Yang, H. D., and Chiou, A. (2017). "Optical-tweezers-based microrheology of soft materials and living cells," in Handbook of Photonics for Biomedical Engineering, eds A. H.-P. Ho, D. Kim, and M. G. Somekh (Dordrecht: Springer), 731-753. doi: 10.1007/978-94007-5052-4_6

Weirich, K. L., Dasbiswas, K., Witten, T. A., Vaikuntanathan, S., and Gardel, M. L. (2019). Self-organizing motors divide active liquid droplets. Proc. Natl. Acad. Sci. U.S.A. 116, 11125-11130. doi: 10.1073/pnas.1814854116

Wieczorek, A., Rezaei, N., Chan, C. K., Xu, C., Panwar, P., Bromme, D., et al. (2015). Development and characterization of a eukaryotic expression system for human type II procollagen. BMC Biotechnol. 15:112. doi: 10.1186/s12896-015-0 228-7

Wu, J., Li, P., Dong, C., Jiang, H., Bin, X., Gao, X., et al. (2018). Rationally designed synthetic protein hydrogels with predictable mechanical properties. Nat. Commun. 9:620. doi: 10.1038/s41467-018-02917-6

Xu, Z., Schaedel, L., Portran, D., Aguilar, A., Gaillard, J., Marinkovich, M. P., et al. (2017). Microtubules acquire resistance from mechanical breakage through intralumenal acetylation. Science 356, 328-332. doi: 10.1126/science.aai8764

Yasuda, R., Miyata, H., and Kinosita, K. (1996). Direct measurement of the torsional rigidity of single actin filaments. J. Mol. Biol. 263, 227-236. doi: 10.1006/jmbi.1996.0571

Yu, H., Dee, D. R., and Woodside, M. T. (2013). Single-molecule approaches to prion protein misfolding. Prion 7, 140-146. doi: 10.4161/pri.23303

Conflict of Interest: The authors declare that the research was conducted in the absence of any commercial or financial relationships that could be construed as a potential conflict of interest.

Copyright (C) 2020 Lehmann, Shayegan, Blab and Forde. This is an open-access article distributed under the terms of the Creative Commons Attribution License (CC BY). The use, distribution or reproduction in other forums is permitted, provided the original author(s) and the copyright owner(s) are credited and that the original publication in this journal is cited, in accordance with accepted academic practice. No use, distribution or reproduction is permitted which does not comply with these terms. 